\title{
On the Existence of Solution of a System of Partial Differential Equations
}

\author{
A. A. Andrian
}

A bstract. Let $\Pi_{\alpha}=\{t \mid 0<\arg t<\alpha\}$ for $\alpha<\pi$ and denote by $M$ the class of $m$-dimensional vector functions $u=u(x, t)$ of $C^{\infty}\left(\mathbb{R}^{n} \times \bar{\Pi}_{\alpha}\right)$ analytic in $t \in \Pi_{\alpha}$ and having polynomial growth in $(x, t)$. Let $A(\xi)\left(\xi \in \mathbb{R}^{n}\right)$ be a square matrix of order $m$ with polynomial elements. In the paper we define regularity and strictly regularity of the system $\frac{\partial u}{\partial t}=A\left(D_{x}\right) u+f$ and prove its solvability in $M$ for all $f \in M$.

Keywords: Partial differential equations, regularity, strictly regularity, solvability AMS subject classiflcation: 35A99

\section{Introduction}

In the following let $\mathbb{N}$ be the set of natural numbers, $N_{0}=\mathbb{N} \cup\{0\}, \Pi$ a complex plane and, for $0<\alpha<\pi$,

$$
\Pi_{\alpha}=\{\lambda \mid 0<\arg \lambda<\alpha\} \quad \text { and } \quad \Pi_{\alpha}^{*}=\left\{\lambda \mid \frac{1}{2} \pi \leq \arg \lambda \leq \frac{3}{2} \pi-\alpha\right\} .
$$

Let $A(\xi) \quad\left(\xi \in \mathbb{R}^{n}\right)$ be a square matrix of order $m$ with polynomial elements such that, for all $\xi \in \mathbb{R}^{n}$, the roots $\lambda_{1}(\xi), \ldots, \lambda_{m}(\xi)$ (some of them can coincide) of the characteristic equation $\operatorname{det}\left(\lambda E_{m}-A(\xi)\right)=0$ satisfy the conditions

$$
\begin{aligned}
\lambda_{1}(\xi), \ldots, \lambda_{r}(\xi) & \in \Pi_{\alpha}^{*} \\
\lambda_{r+1}(\xi), \ldots, \lambda_{m}(\xi) & \in C \Pi_{\alpha}^{*}:=\Pi \backslash \Pi_{\alpha}^{*}
\end{aligned}
$$

where $E_{m}$ is the unit matrix of order $m$. The class $M$ is defined as the set of vector functions

$$
u(x, t)=\left(u_{1}(x, t), \ldots, u_{m}(x, t)\right) \quad \text { of } C^{\infty}\left(\mathbb{R}^{n} \times \bar{\Pi}_{\alpha}\right)
$$

analytic in $t \in \Pi_{\alpha}$ and satisfying the inequality

$$
\left|D_{x}^{j} D_{t}^{k} u(x, t)\right| \leq c_{j k}(1+|x|)^{\gamma}(1+|t|)^{\beta} \quad\left(|j|, k \dot{\in} \mathbb{N}_{0}\right)
$$

for all $(x, t) \in \mathbb{R}^{n} \times \bar{\Pi}_{\alpha}$, where

$$
j=\left(j_{1}, \ldots, j_{n}\right), \quad|j|=j_{1}+\ldots+j_{n}
$$

A. A. Andrian: Armenian State Engin. Univ., Dept. Math., Terian str. 105, 375009 Yerevan, Armenia 
and

$$
D_{x}^{j}=\left(i \frac{\partial}{\partial x_{1}}\right)^{j_{1}} \cdots\left(i \frac{\partial}{\partial x_{n}}\right)^{j_{n}}
$$

Here $i=\sqrt{-1}, D_{t}=\frac{\partial}{\partial t}$ denotes the complex analysis differentiation, $c_{j k}$ are nonnegative constants and $\gamma, \beta \in \mathbb{R}$.

If in (1.2) $\gamma$ is fixed, then the corresponding class we shall denote by $M_{\gamma} \subset M$, and if $u(x, t) \equiv u(t)$, then we have the classes $N$ or $N_{\beta}$.

In the paper the problem of solvability of the system

$$
\frac{\partial u(x, t)}{\partial t}=A\left(D_{x}\right) u(x, t)+f(x, t) \quad\left((x, t) \in \mathbb{R}^{n} \times \Pi_{\alpha}\right)
$$

with unknown $u \in M$ and given $f \in M$ is considered.

The system (1.3) satisfying condition (1.1) will be called strictly regular and the number $r$ will be called its order of regularity. When the condition (1.1) is violated only in a finite number of points, then the system (1.3) is said to be regular.

The main result of the paper looks as follows.

Theorem 1.1. Consider the system (1.9). We have the following statements: $f \in M$.

a) The system (1.9) (regular or strictly regular) admits a solution $u \in M$ for all

b) If the system (1.9) is strictly regular and $f \in M_{\gamma}$, then for its solution $u$ the inclusion $u \in M_{\gamma}$ is true.

c) If the system (1.9) is regular and $f \in M_{\gamma}$, then there exists $\gamma_{1} \geq \gamma$ such that for its solution $u$ the inclusion $u \in M_{\gamma_{1}}$ is true.

Boundary value problems for system (1.3) with $f \equiv 0, t \in \mathbb{R}^{+}=\{t \mid t>0\}$ are studied in $[2,3]$ and for $t \in \Pi_{\alpha}$ in [1]. It should be noted that the operators which are not regular in the sence of $[2,3]$ can become regular in our case $\left(t \in \Pi_{\alpha}\right)$. Par example the Helmholtz operator $\Delta+k^{2}$ with $k>0$, where $\Delta=\frac{\partial^{2}}{\partial t^{2}}+\frac{\partial^{2}}{\partial x^{2}}$ is the Laplacean. Indeed, we have $\lambda_{1,2}(\xi)= \pm \sqrt{\xi^{2}-k^{2}}$ which implies $r=2$ for $|\xi| \leq k$ and $r=1$ for $|\xi|>k$. Now, by taking $t \in \Pi_{\alpha} \quad\left(0<\alpha<\frac{\pi}{2}\right)$ one can see that $r=1$ for all $\xi \neq \pm k$. Another motivation for taking $t \in \Pi_{\alpha}$ is the fact that the solutions of many boundary value problems in reality. can be extended analytically with respect to $t$ to some angle: Par example the solution $u=u(x, t)$ of the Dirichlet problem $u(x, 0)=f(x)$ in the half plane $\mathbb{R} \times \mathbb{R}^{+}$for the Laplace operator is analytic in $t$ from the set $\left\{t|| \arg t \mid<\frac{\pi}{2}\right\}$.

\section{Some auxiliary propositions}

Denote by $F_{\theta}(p)$ the Laplace transform of a function $f(t)$ from the class $N$ when arg $t=$ $\theta$, i.e.

$$
F_{\theta}(p)=\int_{0}^{\infty} e^{-p r} f\left(\tau e^{i \theta}\right) d \tau
$$

We have the following statement. 
Lemma 2.1. The function $F_{0}(p)$ being analytic in the half plane Rep $>0$ extends analytically to the domain $C \Pi_{\alpha}^{*}$.

Proof. From Cauchy integral theorem there follows

$$
\int_{\Gamma_{0} \cup \Gamma_{0}} e^{-p t} f(t) d t=0 \quad\left(\arg p=-\frac{\alpha}{2}\right)
$$

where $\Gamma_{\beta}$ denotes the ray arg $t=\beta, 0 \leq \beta \leq \alpha$. Rewrite (2.1) in the form

$$
F_{0}(p)=e^{i \alpha} F_{\alpha}\left(p e^{i \alpha}\right) \quad\left(\arg p=-\frac{\alpha}{2}\right)
$$

The functions $F_{0}(p)$ and $F_{\alpha}\left(p e^{i \alpha}\right)$ being the Laplace transforms of functions of polynomial growth are analytic in $\operatorname{Re} p>0$ and $\operatorname{Re}\left(p e^{i \alpha}\right)>0$, respectively. So the function

$$
\Phi(p)= \begin{cases}F_{0}(p) & \text { if } \operatorname{Re} p>0 \\ e^{i \alpha} F_{\alpha}\left(p e^{i \alpha}\right) & \text { if } \operatorname{Re}\left(p e^{i \alpha}\right)>0\end{cases}
$$

is analytic in $C \Pi_{\alpha}^{*}$

From the representation

$$
f(t)=P_{s}(t)+g(t) \quad \text { with } P_{s}(t)=f(0)+t f^{\prime}(0)+\ldots+\frac{t^{s}}{s !} f^{(s)}(0)
$$

for any function $f \in N$ and number $s \in N$ we deduce that $g \in N$ and $g^{(j)}(0)=0$ for any $j \leq s$. Let $\beta \in \mathbb{N}$ be such that

$$
\left|D_{t}^{j} f(t)\right|+\left|D_{t}^{j} g(t)\right| \leq c_{j}(1+|t|)^{\beta} \quad\left(t \in \bar{\Pi}_{\alpha}\right) .
$$

Introduce the function

$$
q(t)=g(t)(1+t)^{-2-\beta} \quad\left(q^{(j)}(0)=0 \text { for all } j \leq s\right) .
$$

Now, if $Q(p)$ is the Laplace transform of $q(t)\left(t \in \mathbb{R}^{+}\right) ;$then thanks to the relations (2.3) and (2.4) we have

$$
|Q(p)| \leq c(1+|p|)^{-s-1} \quad\left(p \in C \bar{\Pi}_{\alpha}^{*}\right)
$$

Let $\gamma_{e}=\gamma_{0}+\varepsilon e^{-i \alpha / 2}$, where $\gamma_{0}$ is the boundary of $\Pi_{\alpha}^{*}$ and $\varepsilon>0$. Using the estimation (2.5) it is easy to get

$$
q(t)=\frac{1}{2 \pi i} \int_{e-i \infty}^{e+i \infty} e^{p t} Q(p) d p=\frac{1}{2 \pi i} \int_{\gamma_{0}} e^{p t} Q(p) d p \quad\left(t \in \mathbb{R}^{+}\right)
$$

Evidently this function $q(t)$ is analytic in $\Pi_{\alpha}$ and bounded in $\bar{\Pi}_{\alpha}$. Finally we obtain the following 
Lemma 2.2. Every function $f \in N$ can be presented in the form

$$
f(t)=P_{s}(t)+\frac{(1+t)^{\beta+2}}{2 \pi i} \int_{\gamma_{0}} e^{p t} Q(p) d p \quad\left(t \in \Pi_{\alpha}\right) .
$$

Now let us consider the differential equation

$$
\frac{\partial u(t)}{\partial t}-\lambda u(t)=f(t) \quad\left(t \leq \Pi_{\alpha}\right)
$$

where $\lambda \in \Pi, f \in N$ is given and $u \in N$ is the unknown function. When $t \in \mathbb{R}^{+}$, then the solution of equation (2.6) can be written immediately in function of $\operatorname{sign}(\operatorname{Re} \lambda)$. In our case the form of the solution depends not only of $\lambda \in \Pi_{\alpha}^{*}$ or $\lambda \in C \Pi_{\alpha}^{*}$, but also of a position of $\lambda \dot{\epsilon} C \Pi_{\alpha}^{*}$. For applications we prefer to obtain a unique form of solution.

At first, if $f(t)=P_{s}(t)$ (see (2.2)), then evidently equation (2.6) admits a polynomial solution $u_{0}$. So it remains to consider equation (2.6). with $f=g$ and $\lambda \neq 0$. It is clear that the function

$$
u_{1}(t)=\frac{1}{2 \pi i} \int_{\gamma_{e}} e^{p t} \frac{C_{s}(p)}{p-\lambda} d p \quad\left(t \in \Pi_{\alpha}\right)
$$

where $\varepsilon$ is such that $\lambda$ and 0 are placed on different sides of $\gamma_{\epsilon}$ in the case of $\lambda \in C \Pi_{\alpha}^{*}$, is a solution of equation (2.6) satisfying the estimate $\left|u_{1}(t)\right| \leq c e^{e|t|}\left(t \in \bar{\Pi}_{\alpha}\right)$. Let us show that $u_{1} \in N$. Since $f \in N$ and $u_{1}$ satisfies equation (2.6) it is sufficient to verify inequality (1.2) only for $k=0$. From (2.4) we have $G(p)=\left(1-\frac{d}{d p}\right)^{\beta+2} Q(p)$. Then by integrating by parts we get

$$
\begin{aligned}
u_{1}(t) & =\frac{1}{2 \pi i} \int_{\gamma_{c}} \frac{e^{p t}}{p-\lambda}\left(1-\frac{d}{d p}\right)^{\beta+2} Q(p) d p \\
& =\frac{1}{2 \pi i} \int_{\gamma_{0}} Q(p)\left(1+\frac{d}{d p}\right)^{\beta+2}\left(\frac{e^{p t}}{p-\lambda}\right) d p \\
& =\sum_{j \leq \beta+2} t^{j} \int_{\gamma_{0}} q_{j}(p) Q(p) e^{p t} d p \quad\left(t \in \bar{\Pi}_{\alpha}\right)
\end{aligned}
$$

where the functions $q_{j}(p)$ are well-defined and bounded, i.e. $u_{1}(t)$ is of polynomial growth. Hence the following lemma is proved.

Lemma 2.3. The equation (2.6) admits a solution $u \in N$ for all functions $f \in N$.

Now, if we have a system

$$
\frac{\partial u(t)}{\partial t}-A u(t)=f(t): \quad\left(t \in \Pi_{\alpha}\right)
$$

where $A$ is a constant matrix of order $m, f \in N$ is a given vector function and $u \in N$ is the unknown vector function, we can solve it by transforming the matrix $A$ into Jordan form and using Lemma 2.3 . 


\section{Construction of a solution of system (1.3)}

Strictly regular case. Let the system (1.3) be strictly regular. Introduce the following polynoms in $\lambda$ :

$$
\begin{aligned}
& Q(\xi, \lambda)=\prod_{j=1}^{r}\left(\lambda-\lambda_{j}(\xi)\right)=\lambda^{r}+\sum_{j=1}^{r} a_{j}(\xi) \lambda^{r-j} \\
& R(\xi, \lambda)=\prod_{j=1}^{m-r}\left(\lambda-\lambda_{r+j}(\xi)\right)=\lambda^{m-r}+\sum_{j=1}^{m-r} a_{r+j}(\xi) \lambda^{m-r-j}
\end{aligned}
$$

It is well known (see [4: Lemma 3.1/p. 194]) that

$$
\begin{aligned}
\mid \lambda_{j}(\xi) & \leq c_{j}(1+|\xi|)^{n_{j}}, & & a_{j} \in C^{\infty}\left(\mathbb{R}^{n}\right) \\
\left|D_{\xi}^{k} a_{j}(\xi)\right| & \leq c_{k}(1+|\xi|)^{n_{j k}} & & \left(|k| \in \mathbb{N}_{0}\right) .
\end{aligned}
$$

Let $v(\xi, t)$ be a solution of the Cauchy problem

$$
\begin{aligned}
Q\left(\xi, \frac{\partial}{\partial t}\right) v(\xi, t) & :=\frac{\partial^{r} v(\xi, t)}{\partial t^{r}}+\sum_{j=1}^{r} a_{j}(\xi) \frac{\partial^{r-j} v(\xi, t)}{\partial t^{r-j}}=0 \quad\left(t \in \Pi_{\alpha}\right) \\
D_{\imath}^{j} v(\xi, 0) & =b_{j}(\xi) \quad(0 \leq j \leq r-1)
\end{aligned}
$$

where $b_{j} \in C^{\infty}\left(\mathbb{R}^{n}\right)$ satisfies inequality (3.1) and $v(\xi, t)$ is analytic in $t \in \Pi_{\alpha}$. Rewrite (3.2), (3.3) in matrix form

$$
\begin{aligned}
\frac{\partial \tilde{w}(\xi, t)}{\partial t} & =B(\xi) w(\xi, t) \quad\left(t \in \Pi_{\alpha}\right) \\
\dot{w}(\xi, 0) & =b(\xi)
\end{aligned}
$$

where

$$
w=\left(v, \frac{\partial v}{\partial t}, \ldots, \frac{\partial^{r-1} v}{\partial t^{r-1}}\right) \quad \text { and } \quad b(\xi)=\left(b_{0}(\xi), \ldots, b_{r-1}(\xi)\right)
$$

and $B(\xi)$ is a well-defined square matrix of order $r$ with eigenvalues $\lambda_{1}(\xi), \ldots, \lambda_{r}(\xi)$ all belonging to $\Pi_{\alpha}^{*}$. We have $w(\xi, t)=e^{B(\xi) t} b(\xi)$, whence by using the well-known estimate

$$
\left\|e^{B(\xi) t}\right\| \leq c \sum_{j=1}^{r} e^{\operatorname{Re}\left(\lambda_{j}(\xi) t\right)}(1+|t|)^{r-1}(1+|\xi|)^{s}
$$

(see [2: p. 222, item 2]) we shall get the estimate

$$
\left|D_{\xi}^{j} D_{t}^{k} v(\xi, t)\right| \leq c_{j k}(1+|\xi|)^{m_{j k}}(1+|t|)^{n_{j k}} .
$$

For beginning we take $f(x, t)=t^{j} q(x) \in M$. Every function $u(x, t) \in M$ can be considered as a distribution from $S^{\prime}\left(\mathbb{R}^{n}\right)$ depending on parameter $t \in \bar{\Pi}_{\alpha}$ and satisfying the inequality

$$
\left|\frac{\partial^{j}}{\partial t^{j}}\langle u(x, t), \varphi(x)\rangle\right| \leq c_{j}(1+|t|)^{\beta}\|\varphi\|_{s} \quad\left(\varphi \in S\left(\mathbb{R}^{n}\right)\right)
$$


where $S\left(\mathbb{R}^{n}\right)$ and $S^{\prime}\left(\mathbb{R}^{n}\right)$ are the pair of Schwartz spaces and

$$
\|\varphi\|_{s}=\sup _{x}\left(1+|x|^{2}\right)^{s / 2} \sum_{|\alpha| \leq s}\left|D_{x}^{\alpha} \varphi(x)\right| \quad\left(s \in \mathbb{N}_{0}\right)
$$

Let $\hat{u}(\xi, t)=F_{x}(u(x, t))$ denote the generalized Fourier transform of $u \in M\left(F^{-1}\right.$ will denote the inverse Fourier transform). The Fourier image of system (1.3) has the form

$$
\frac{\partial \hat{u}(\xi, t)}{\partial t}=A(\xi) \hat{u}(\xi, t)+t^{j} \hat{q}(\xi)
$$

As a solution of system (3.5) we consider

$$
\hat{u}(\xi, t)=\frac{j !}{2 \pi i} \int_{\gamma^{-}(\xi)}\left(\lambda E_{m}-A(\xi)\right)^{-1} \lambda^{-j-1} e^{\lambda t} d \lambda \hat{q}(\xi)
$$

where $\gamma^{-}(\xi)$ is a closed contour containing only the roots $\lambda_{1}(\xi), \ldots, \lambda_{r}(\xi) \in \Pi_{\alpha}^{*}$ and the point $\lambda=0$. Introduce the matrix

$$
V(\xi, t)=\frac{j !}{2 \pi i} \int_{\gamma^{-}(\xi)}\left(\lambda E_{m}-A(\xi)\right)^{-1} \lambda^{-j-1} e^{\lambda t} d \lambda \quad\left(t \in \Pi_{\alpha}\right) .
$$

Lemma 3.1. The elements $v_{l s}(\xi, t)$ of the matrix $V(\xi, t)$ satisfy the inequality (9.4).

Proof. We have

$$
V(\xi, 0)=\frac{j !}{2 \pi i} \int_{\gamma^{-}(\xi)} \frac{a(\xi, \lambda)}{\lambda^{j+1} Q(\xi, \lambda) R(\xi, \lambda)} d \lambda
$$

with

$$
a(\xi, \lambda)=\left(a E_{m}-A(\xi)\right)^{-1}\left|\lambda E_{m}-A(\xi)\right| .
$$

Since the polynomials $\lambda^{j+1} Q(\xi, \lambda)$ and $R(\xi, \lambda)$ are coprime, then

$$
1=r(\xi, \lambda) \lambda^{j+1} Q(\xi, \lambda)+q(\xi, \lambda) R(\xi, \lambda)
$$

where $r(\xi, \lambda)$ and $q(\xi, \lambda$ are polynomials in $\lambda$ with coefficients satisfying the inequality (3.1). The matrix $V(\xi, 0)$ can be rewritten in the form

$$
V(\xi, 0)=\frac{j !}{2 \pi i} \int_{\gamma^{-}(\xi)} \frac{q(\xi, \lambda)}{\lambda^{j+1} Q(\xi, \lambda)} a(\xi, \lambda) d \lambda .
$$

Since the contour $\gamma^{-}(\xi)$ encloses all roots of denominator $\lambda^{j+1} Q(\xi, \lambda)$, then computing (3.8) with help of the residue theorem at the point $\lambda=\infty$ we immediately get

$$
\left|D_{\xi}^{k} D_{i}^{j} v_{l s}(\xi, 0)\right| \leq c_{k j}(1+|\xi|)^{n_{k j}} \quad\left(|k|, j \in \mathbb{N}_{0}\right)
$$

Now notice that the function $v_{l s}(\xi, t)$ satisfies the differential equation

$$
\frac{\partial^{j+1}}{\partial t^{j+1}} Q\left(\xi, \frac{\partial}{\partial t}\right) v_{l s}(\xi, t)=0
$$

the characteristic roots of which belong to the set $\Pi_{\alpha}^{*}$. Therefore it satisfies the estimate (3.4) (see (3.2) and (3.3)) 
Lemma 3.2. The inclusion $F_{\xi}^{-1}\left(v_{l s}(\xi, t) \hat{q}_{j}(\xi)\right) \in M$ is true, where the vector function $q(x)=\left(q_{1}(x), \ldots, q_{m}(x)\right)$ is an element of $M$.

Proof. We have $\left|D_{x}^{k} q_{j}(x)\right| \leq c_{k}(1+|x|)^{\gamma}$ (see (1.2)). Let $k_{0} \gg 1$ be such that the function

$$
w_{l s}(x, t)=F_{\xi}^{-1}\left(\left(1+|\xi|^{1}\right)^{-k_{0}} v_{l s}(\xi, t)\right)=\frac{1}{(2 \pi)^{n}} \int_{\mathbb{R}^{n}}\left(1+|\xi|^{2}\right)^{-k_{0}} v_{l s}(\xi, t) e^{-i \xi x} d \xi
$$

satisfies the estimate

$$
\left|w_{l s}(x, t)\right| \leq c(1+|x|)^{-n-|\gamma|-1}(1+|t|)^{m_{0}}
$$

And if $\Delta_{x}=\sum_{j=1}^{n} \frac{\partial^{2}}{\partial x_{j}^{2}}$, then we have

$$
F_{\xi}^{-1}\left(v_{l s}(\xi, t) \hat{q}_{j}(\xi)\right)=w_{l s}(x, t) *\left(1-\Delta_{x}\right)^{k_{0}} q(x)
$$

where the operation $*$ is taken in relation to the variable $x$, and according to the Peetre inequalities

$$
(1+|x-y|)^{-|\gamma|} \leq(1+|x|)^{\gamma}(1+|y|)^{\gamma}
$$

and

$$
(1+|x-y|)^{\gamma} \leq(1+|x|)^{\gamma}(1+|y|)^{\gamma}
$$

when $\gamma \leq 0$ or $\gamma>0$, respectively, we get

$$
\begin{aligned}
\left|F_{\xi}^{-1}\left(v_{l s}(\xi, t) \hat{q}_{j}(\xi)\right)\right| & \leq c(1+|x|)^{\gamma} \cdot(1+|t|)^{m_{0}} \int(1+|y|)^{-n-|\gamma|-1}(1+|y|)^{\gamma} d y \\
& \leq c_{1}(1+|x|)^{\gamma}(1+|t|)^{m_{0}} .
\end{aligned}
$$

The derivatives can be estimated similarly

Thus, if $f(x, t)=t^{j} q(x)$, then the solution $u$ of system (1.3) is constructed, namely

$$
u(x, t)=F_{\xi}^{-1}\left(\frac{j !}{2 \pi i} \int_{\gamma^{-}(\xi)}\left(\lambda E_{m}-A(\xi)\right)^{-1} \lambda^{-j-1} e^{\lambda t} d \lambda \cdot \hat{q}(\xi)\right)
$$

So, using the representation

$$
f(x, t)=f(x, 0)+t \frac{\partial f}{\partial t}(x, 0)+\ldots+\frac{t^{l}}{l !} \frac{\partial^{l} f}{\partial t^{l}}(x, 0)+g(x ; t)
$$

with an arbitrary $l \in \mathbb{N}$, we can reduce our problem to

$$
\frac{\partial u(x, t)}{\partial t}=A\left(D_{x}\right) u(x, t)+g(x, t)
$$


where $g \in M$. and $D_{t}^{j} g(x, 0)=0$ for all $j \leq l$. In (1.2) assume $\beta \in \mathbb{N}$. Introduce the vector function (see (2.4))

$$
q(x, t)=g(x, t)(1+t)^{-\beta-2}
$$

As Fourier image of a solution of system (3.10) we consider the expression

$$
\hat{u}(\xi, t)=\frac{1}{2 \pi i} \int_{\gamma_{\epsilon}(\xi)}\left(\lambda E_{m}-A(\xi)\right)^{-1} \hat{G}(\xi, \lambda) e^{\lambda t} d \lambda
$$

where $\gamma_{\varepsilon}(\xi)=\gamma_{0}+\varepsilon(\xi) e^{-i \alpha / 2}, \varepsilon(\xi)>0$, is such that the roots $\lambda_{r+1}(\xi), \ldots, \lambda_{m}(\xi) \in C \Pi_{\alpha}^{*}$ and the point $\lambda=0$ are on different sides of it (see (2.7)) and $\hat{G}(\xi, \lambda)$ is the LaplaceFourier transform of $g(x, t) \quad\left((x, t) \in \mathbb{R}^{n} \times \mathbb{R}^{+}\right)$.

A formula analogous to (3.7) permits us to rewrite (3.12) in the form

$$
\begin{aligned}
\hat{u}(\xi, t)= & \frac{1}{2 \pi i} \int_{\gamma_{\epsilon}(\xi)} \frac{q(\xi, \lambda) a(\xi, \lambda)}{Q(\xi, \lambda)} \hat{G}(\xi, \lambda) e^{\lambda t} d \lambda \\
& +\frac{1}{2 \pi i} \int_{\gamma_{\epsilon}(\xi)} \frac{r(\xi, \lambda) a(\xi, \lambda)}{R(\xi, \lambda)} \hat{G}(\xi, \lambda) e^{\lambda t} d \lambda \quad\left(t \in \Pi_{\alpha}\right) .
\end{aligned}
$$

The integrals here are convergent because of $D_{t}^{j} g(x, 0)=0$ for any $j \leq l$ with $l \gg 1$ taken in advance.

Let $l_{0}$ be such that

$$
\left\|\frac{q(\xi, \lambda) a(\xi, \lambda)}{\lambda^{I_{0}} Q(\xi, \lambda)}\right\| \leq c(\xi) \frac{1}{|\lambda|} \quad(|\lambda|>1) .
$$

Introduce the matrix

$$
\hat{K}(\xi, t)=\frac{1}{2 \pi i} \int_{\gamma^{-}(\xi)} \frac{q(\xi, \lambda) a(\xi, \lambda)}{\lambda^{l_{0}} Q(\xi, \lambda)} e^{\lambda t_{0}} d \lambda
$$

with $\gamma^{-}(\xi)$ defined in (3.6). The first integral in (3.13) will take the form

$$
\hat{u}_{1}(\xi, t)=\int_{0}^{t} \hat{K}(\xi, \tau) \hat{g}_{t}^{\left(l_{0}\right)}(\xi, t-\tau) d \tau \quad\left(t \in \Pi_{\alpha}\right)
$$

As it was shown above (see Lemma 3.1 ) the elements $\hat{k}_{l s}(\xi, t)$ of the matrix $\hat{K}(\xi, t)$ satisfy the estimation (3.4), hence we can easily regularize the inverse transform $u_{1}(x, t)$ of $\hat{u}_{1}(\xi, t)$ (see Lemma 3.2) and have the inclusion $u_{1} \in M$.

Now let us examine the second term $\hat{u}_{2}(\xi, t)$ in (3.13). At first as

$$
\hat{G}(\xi, t)=\left(1-\frac{d}{d \lambda}\right)^{\beta+2} \hat{Q}(\xi, \lambda)
$$


(see (3.11)), where $\hat{Q}(\xi, \lambda)$ satisfies an inequality like (2.5), integrating by parts we can reduce $\hat{u}_{2}(\xi, t)$ to the form

$$
\hat{u}_{2}(\xi, t)=\frac{1}{2 \pi i} \int_{\gamma_{0}} \hat{Q}(\xi, \lambda) \psi(\xi, \lambda, t) d \lambda \quad\left(t \in \Pi_{\alpha}\right)
$$

where

$$
\psi(\xi, \lambda, t)=\left(1+\frac{d}{d \lambda}\right)^{\beta+2} \frac{r(\xi, \lambda) a(\xi, \lambda) e^{\lambda t}}{R(\xi, \lambda)}
$$

As consequence of the inequality

$$
|R(\xi, \lambda)| \geq c\left(1+|\xi|^{2}\right)^{s} \quad\left((\xi, \lambda) \in \mathbb{R}^{n} \times \gamma_{0}\right)
$$

(see [1: Lemma 1.2]) we get the estimation

$$
\left|D_{\xi}^{k} \psi(\xi, \lambda, t)\right| \leq c_{k}(1+|\xi|)^{m_{k}}(1+|\lambda|)^{n_{k}}(1+|t|)^{\beta+2}
$$

for all $(\xi, \lambda, t) \in \mathbb{R}^{n} \times \gamma_{0} \times \Pi_{\alpha}$. Now it is clear that by the same way

$$
u_{2}(x, t)=F_{\xi}^{-1}\left(\hat{u}_{2}(\xi, t)\right)=\frac{1}{2 \pi i} F_{\xi}^{-1}\left(\int_{\gamma_{0}} \hat{Q}(\xi, \lambda) \psi(\xi, \lambda, t) d \lambda\right) \in M
$$

Theorem 1 for strictly regularity of system (1.3) is proved.

2. Regular case. In the considerations made above the condition (1.1) (condition of strictly regularity) was very essential (see estimation (3.1) and others). Assuming that the condition (1.1) is violated only at the point $\xi=0$ (this does not loss the generality) we can show (see [1: Lemmas 1.2 and 1.3]) that in all inequalities crucial (i.e. in (3.1), (3.14) and (3.15)) we shall have the factor $|\xi|^{s_{k}}$ with $s_{k} \leq 0$ and this circumstance adds new difficulties (the estimation (3.9) was established thanks to smoothness of $v_{l s}(\xi, t)$ in $\xi \in \mathbb{R}^{n}$ ).

Let us demonstrate how we can surmount this obstacle. First, consider the equation $\Delta_{x} u(x, t)=f(x, t)$, where $\Delta_{x}$ is the Laplacean, $f \in M$ is a given function and $u \in M$ is the unknown function, and let us prove its solvability. If $n=1$, i.e. $\Delta_{x}=\frac{\partial^{2}}{\partial x^{2}}$, then obviously this equation admits a solution from $M$. Now we are going to show how the case $n \geq 2$ can be reduced to the case $n-1$. For this purpose we replace $f(x, t)$ with $\left(i \frac{\partial}{\partial x_{1}}\right)^{v_{1}} f(x, t)$ and consider the expression $|\xi|^{-2} \xi_{1}^{v_{1}} \hat{f}(\xi, t)$. With an appropriate choice of natural $v_{1}$ we can reach any smothness order in $\mathbb{R}^{n}$ of the function $|\xi|^{-2} \xi_{1}^{v_{1}}$. By taking a natural $k \gg 1$ we can readly establish the inclusion

$$
\begin{aligned}
u_{0}(x, t) & =F_{\xi}^{-1}\left(|\xi|^{-2} \xi_{1}^{v_{1}}\left(1+|\xi|^{2}\right)^{-k}\left(1+|\xi|^{2}\right)^{k} \hat{f}(\xi, t)\right) \\
& =F_{\xi}^{-1}\left(|\xi|^{-2} \xi_{1}^{v_{1}}\left(1+|\xi|^{2}\right)^{-k}\right) *\left(1-\Delta_{x}\right)^{k} f(x, t) \\
& \in M
\end{aligned}
$$


where the operation * is taken in relation to the variable $x$ and $\Delta_{x} u_{0}=\left(i \frac{\partial}{\partial x_{1}}\right)^{v_{1}} f$. Find $u_{1} \in M$ in a way that $\left(i \frac{\partial}{\partial x_{1}}\right)^{v_{1}} u_{1}=u_{0}$. Then we get

$$
\left(i \frac{\partial}{\partial x_{1}}\right)^{v_{1}}\left(\Delta_{x} u_{1}-f\right)=0
$$

or, by integrating with respect to $x_{1}$,

$$
\Delta_{x} u_{1}=f+\sum_{j \leq v_{1}-1} a_{j}\left(x^{\prime}, t\right) x_{1}^{j}, \quad x^{\prime}=\left(x_{2}, \cdots, x_{n}\right),
$$

where $a_{j}\left(x^{\prime}, t\right) \in M$ are well-defined functions. Introducing $w=u_{1}-u$ we shall get

$$
\Delta_{x} \dot{w}=\sum_{j \leq v_{1}-1} a_{j}\left(x^{\prime}, t\right) x_{1}^{j}
$$

Let us seek $w(x, t)$ in the form

$$
w(x, t)=\sum_{j \leq v_{1}-1} c_{j}\left(x^{\prime}, t\right) x_{1}^{j}
$$

with unknown $c_{j}\left(x^{\prime}, t\right) \in M$. Substituting $w(x, t)$ into equation (3.16) and making use of linearly independence of the functions $1, x_{1}, \ldots, x_{1}^{v_{1}-1}$, we obtain

$$
\begin{aligned}
\Delta_{x^{\prime}} c_{v_{1}-1}\left(x^{\prime}, t\right) & =a_{v_{1}-1}\left(x^{\prime}, t\right) \\
\Delta_{x^{\prime}} c_{j}\left(x^{\prime}, t\right) & =B_{j}\left(c_{j+1}\left(x^{\prime}, t\right), \ldots, c_{v_{1}-1}\left(x^{\prime}, t\right)\right)+b_{j}\left(x^{\prime}, t\right) \\
j & =v_{1}-2, \ldots, 0
\end{aligned}
$$

where $B_{j}(\cdots)$ is a linear expression and $b_{j}\left(x^{\prime}, t\right) \in M$ are certain functions. Thus the desired reduction is realized. Hence we have proved the following

Lemma 3.3. The equation $\Delta_{x} u=f$ is always solvable in $M$.

The solvability of the system (1.3) can be established in the same way: first in (1.3) we put $\Delta_{x}^{v} f(x, t)$ instead of $f(x, t)$ and with an appropriate choice of natural $v$ we get solvability of the system

$$
\frac{\partial u}{\partial t}=A\left(D_{x}\right) u+\Delta_{x}^{v} f
$$

Further, let $u_{0}$ denote a solution of system (3.17). Then according to Lemma 3.3 we can find $u_{1} \in M$ in a way that $\Delta_{x}^{v} u_{1}=u_{0}$. Now we put $u_{0}$ in (3.17). and get

$$
\Delta_{x}^{v}\left(\frac{\partial u_{1}}{\partial t}-A\left(D_{x}\right) u_{1}-f\right)=0
$$

With the help of Fourier transformation from (3.18) we deduce

$$
\frac{\partial u_{1}}{\partial t}=A\left(D_{x}\right) u_{1}+f+\sum_{|j| \leq 2 v-1} a_{j}(t) x^{j}
$$


with certain coefficients $a_{j} \in M$. Now consider the system

$$
\frac{\partial w}{\partial t}=A\left(D_{x}\right) w+\sum_{|j| \leq 2 v-1} a_{j}(t) x^{j}
$$

and let us seek $w \in M$ in the form

$$
w=\sum_{|j| \leq 2 v-1} c_{j}(t) x^{j}
$$

with unknown coefficients $c_{j} \in N$. Substituting $w$ into (3.19) and taking account of linearly independence of polynoms $\left\{x^{j}|| j \mid=0, \ldots, 2 v-1\right\}$ we get the system

$$
\frac{\partial c_{j}(t)}{\partial t}=A(0) c_{j}(t)+a_{j}(t) \quad(|j|=2 v-1)
$$

which is solvable (see the system (2.9)). The rest $c_{j}(|j| \leq 2 v-2)$ can be determined in the same manner. Evidently $u_{1}-w$ is the desired solution of system (1.3). Theorem 1 is completely proved.

\section{References}

[1] Andrian, A. A.: A general boundary value problem in dihedral domain for systems of partial differential equations (in Russian). Izvestiya Akademii Nauk Armenii, Matematika 28 (1993)2, 1 - 18.

[2] Shilov, G. E.: Mathematical Analysis. Second Special Course (in Russian). Moscow: Nauka 1965.

[3] Tovmasian, N. E.: Correct boundary value problems in half-space for a system of partial differential equations in a class of functions with polynomial growth. Izvestiya A kademii Nauk Arm. SSR, Matematika 23 (1988), 309 - 324.

[4] Treves, F.: Introduction to Pseudodifferential and Fourier Integral Operators. Vol. I. New York and London: Plenum Press 1980.

Received 13.08.1992; in revised form 11.04.1994 